\title{
Erich Lehmann's Contributions to Orderings of Probability Distributions
}

\author{
Marco Scarsini
}

The initial contributions to the theory of comparison of experiments appeared in the late forties/early fifities (see, e.g., Bohnenblust, Shapley, and Sherman,, 1949; Stein, 1951; Blackwell, 1951, 1953).

At around the same time, motivated by some problems in hypothesis testing, Erich Lehmann introduced some basic concepts in the theory of comparison of distributions. As later developments show, the two theories are strictly connected and they often examine conditions on two probability measures $P$ and $Q$ under which $\int \phi \mathrm{d} P \leqslant$ $\int \phi \mathrm{d} Q$ for all functions $\phi$ in some class.

The success of the concept has been enormous both in probability theory and in applications in statistics, decision theory, economics, operations research, and reliability theory. We refer to Müller and Stoyan (2002); Shaked and Shanthikumar (2007) for an extensive analysis of stochastic orders and their applications.

\section{Testing multiparameter hypotheses}

Lehmann (1952) is the first paper where a monotonicity condition is considered, in the framework of hypothesis testing. The hypothesis studied in this paper concerns different parameters. It could be, for instance, of the type $H: \theta_{1} \leqslant \theta_{1}^{*}, \theta_{2} \leqslant \theta_{2}^{*}$. For this type of hypotheses an unbiased test does not exist in general.

Therefore a restriction of monotonicity is imposed, that is, the rejection region for $H$ is assumed to be an increasing set. This makes sense when for $\theta_{1} \leqslant \theta_{1}^{\prime}$ and $\theta_{2} \leqslant \theta_{2}^{\prime}$ we have that $F_{\theta_{1} \theta_{2}}$ is stochastically dominated by $F_{\theta_{1}^{\prime} \theta_{2}^{\prime}}$. The uniformly most powerful test among all monotonic regression regions is found and it has a simple natural form.

M. Scarsini

Dipartimento di Economia e Finanza, LUISS, Viale Romania 12, I-00197 Roma, Italy

e-mail: marco.scarsini@luiss.it 


\section{Ordered families of distributions}

Lehmann (1955) introduces what is now known as the usual stochastic order and the monotone likelihood ratio order.

Using the same notation as in Lehmann's paper, two distributions $P_{\theta}$ and $P_{\theta^{\prime}}$ can be compared for instance according to the following four criteria:

(i) There exists a random vector $Z$ and two functions $g, g^{\prime}$ such that $g(Z)$ has distribution $P_{\theta}, g^{\prime}(Z)$ has distribution $P_{\theta^{\prime}}$ and $g(z) \leqslant g^{\prime}(z)$ for all $z$.

(ii) For every increasing function $\phi$,

$$
\mathbb{E}_{\theta} \phi(X) \leqslant \mathbb{E}_{\theta^{\prime}} \phi(X) .
$$

(iii) For every increasing set $S$,

$$
P_{\theta}(S) \leqslant P_{\theta^{\prime}}(S) .
$$

(iv) Let $P_{\theta}$ and $P_{\theta^{\prime}}$ have densities $p_{\theta}$ and $p_{\theta^{\prime}}$ with respect to some common measure $\mu$. Then the ratio $p_{\theta^{\prime}}(x) / p_{\theta}(x)$ is increasing.

The three conditions (i), (ii), (iii) are equivalent and this has been proved later in great generality by Kamae, Krengel, and O'Brien (1977) using results by Strassen (1965). Lehmann (1955) shows the equivalence of (ii) and (iii) and provides two counterexamples (Examples 2.2 and 2.3 in the paper) to the equivalence of (i) and (ii). Unfortunately there is a mistake, since the two distributions used in the counterexample are actually not ordered.

Condition (iv) was studied extensively by Karlin and Rubin (1956a,b). For univariate distributions this condition is stronger than (i), but for multivariate distributions this is not the case, as Example 2.4 rightly shows. The sufficient multivariate condition that implies (i) is the following

$$
p_{\theta^{\prime}}(x) p_{\theta}(y) \leqslant p_{\theta}(x \wedge y) p_{\theta^{\prime}}(x \vee y),
$$

where $\wedge$ and $\vee$ indicate the componentwise minimum and maximum, respectively. This condition was studied by Karlin and Rinott (1980) and Whitt (1982).

\section{Comparing location experiments}

Lehmann (1988) starts with a review of the classical theory of comparison of experiments developed in the late forties by several scholars and culminated with Blackwell $(1951,1953)$. We refer to the book by Torgersen (1991) and to the nice survey by Le Cam (1996). 
An experiment $\mathscr{E}$ is defined as $\{X, \mathscr{P}\}$, where $X$ is a random quantity and $\mathscr{P}=$ $\left\{P_{\theta}\right.$,

$\theta \in \Omega\}$ is a parametric family of possible distributions of $X$.

Call $\mathscr{F}=\left\{Y, \mathscr{Q}=\left\{Q_{\theta}, \theta \in \Omega\right\}\right\}$ another experiment where $P_{\theta}$ and $Q_{\theta}$ are indexed by the same state of nature. The experiment $\mathscr{F}$ is more informative than the experiment $\mathscr{E}$ if there exists a random quantity $Z$ with known distribution and a function $h$ such that for all $\theta \in \Omega$

$Y$ is distributed as $Q_{\theta}$ implies that $h(Y, Z)$ is distributed as $P_{\theta}$.

Blackwell proved that $\mathscr{F}$ is more informative than $\mathscr{E}$ if an only if for any decision procedure based on $X$ and any loss function there exists a (possibly randomized) decision procedure based on $Y$ that guarantees a weakly smaller risk.

Some examples show that the above criterion is too demanding and does not allow to compare experiments that should be intuitively comparable. Lehmann (1988) therefore suggests a weaker criterion that involves considering only a sub-class of decision problems, in particular the class $\mathscr{M}$ of monotone procedures introduced by Karlin and Rubin (1956b). In these procedures the correct action is a nondecreasing function $\alpha(\theta)$ and the loss function $L(\theta, d)$ is nondecreasing in $d-\alpha(\theta)$ for $d-\alpha(\theta)>0$ and nonincreasing in $d-\alpha(\theta)$ for $d-\alpha(\theta)<0$, with $L(\theta, \alpha(\theta))=0$.

Define $F_{\theta}(t)=P_{\theta}(X \leqslant t)$ and $G_{\theta}(t)=Q_{\theta}(Y \leqslant t)$, and let $F_{\theta}$ and $G_{\theta}$ have densities with monotone likelihood ratio. The main theorem states that $Y$ is more effective than $X$ with respect to the class $\mathscr{M}$ if and only if $h_{\theta}(t)=G_{\theta}^{-1}\left(F_{\theta}(t)\right)$ is nondecreasing in $\theta$ for each $t$. This condition corresponds to the comparison of the two P-P plots $\left(F_{\theta_{1}}, F_{\theta_{2}}\right)$ and $\left(G_{\theta_{1}}, G_{\theta_{2}}\right)$, for $\theta_{1}<\theta_{2}$, and, unlike Blackwell's condition, is easy to verify.

For location families with strongly unimodal densities the condition of the theorem reduces to domination in term of the "more spread out" order studied by Bickel and Lehmann (1979).

This idea has found extensive application in the economic literature (see, for instance, Persico, 2000; Levin, 2001; Athey and Levin, 2001; Bergemann and Välimäki, 2002; Quah and Strulovici, 2009, and references therein). In particular Jewitt (2007) examines conditions under which the Lehmann criterion is applicable to various economic problems.

\section{Invariant directional orderings}

Several stochastic orderings have been studied and used since Lehmann (1955). Some are stronger than others. For instance, the likelihood ratio order is stronger than the hazard order, which in turn is stronger than the usual stochastic order. These three orders are defined in totally different ways and the above chain of implications is not immediate. Lehmann and Rojo (1992) provide a unified way to define these three orders and many others. 
The paper considers a class $\mathscr{F}$ of continuous strictly increasing distribution functions and a preorder $S \subset \mathscr{F} \times \mathscr{F}$.

A preorder $S$ is invariant under monotone transformations if $(F, G) \in S$ implies $\left(F \psi^{-1}, G \psi^{-1}\right) \in S$ for all $\psi \in \Psi$, the class of strictly increasing continuous functions. If $S$ is invariant under monotone transformations and $(F, G) \in S$, then $\mathscr{O}(F, G) \subset S$, where

$$
\mathscr{O}(F, G)=\left\{\left(F \psi^{-1}, G \psi^{-1}\right): \psi \in \Psi\right\}
$$

is the orbit of $(F, G)$ under the group of transformations $\Psi$.

Each orbit $\mathscr{O}(F, G)$ can be labeled by the function $k:[0,1] \rightarrow[0,1]$ defined as $k(u)=G F^{-1}(u)$. Different orderings can be characterized through properties of the function $k$. So a preorder $S$ can be defined as

$$
S=\{(F, G): k(F, G) \in \mathscr{K}\}
$$

for some class $\mathscr{K}$ that contain the identity function and is closed under composition. Different choices of $\mathscr{K}$ produce the likelihood ratio order, the hazard rate order, and the usual stochastic order.

This approach allows even to order ordered pairs. Let $\left(F_{1}, G_{1}\right),\left(F_{2}, G_{2}\right) \in S$. Then $G_{2}$ is said to be further to the right of $F_{2}$ than $G_{1}$ is of $F_{1}$ if $\left(k_{1}, k_{2}\right) \in S$, where $k_{i}=G_{i} F_{i}^{-1}$.

The paper also defines metrics that are coherent with the various orders, that is, metrics $d_{S}$ that are invariant with respect to monotone transformations and such that $d_{S}\left(F_{1}, G_{1}\right) \leqslant d_{S}\left(F_{2}, G_{2}\right)$ whenever $\left(k_{1}, k_{2}\right) \in S$.

The technique discovered in this paper has been used by Bartoszewicz and Benduch (2009) to define orders based on a generalization of the total time on test transform.

\section{References}

Athey, S. and Levin, J. (2001) The Value of Information in Monotone Decision Problems. Working Paper 98-24, MIT.

BArtoszewicz, J. and Benduch, M. (2009) Some properties of the generalized TTT transform. J. Statist. Plann. Inference 139, 2208-2217.

BERGEMANN, D. and VÄLIMÄKI, J. (2002) Information acquisition and efficient mechanism design. Econometrica 70, 1007-1033.

BICKEL, P. J. and LEHMANN, E. L. (1979) Descriptive statistics for nonparametric models. IV. Spread. In Contributions to statistics, 33-40. Reidel, Dordrecht.

Blackwell, D. (1951) Comparison of experiments. In Proceedings of the Second Berkeley Symposium on Mathematical Statistics and Probability, 1950, 93-102. University of California Press, Berkeley and Los Angeles.

BLACKWELl, D. (1953) Equivalent comparisons of experiments. Ann. Math. Statistics 24, 265-272.

Bohnenblust, H. F., Shapley, L. S., and Sherman, S. (1949) Reconnaissance in game theory. Technical Report RM 208, RAND Corporation, Santa Monica, CA. URL http://www.rand.org/ pubs/research_memoranda/RM208. 
JEWITT, I. (2007) Information order in decision and agency problems. Mimeo, Nuffield College.

Kamae, T., Krengel, U., and O'Brien, G. L. (1977) Stochastic inequalities on partially ordered spaces. Ann. Probability 5, 899-912.

KARLIN, S. and RinOTT, Y. (1980) Classes of orderings of measures and related correlation inequalities. I. Multivariate totally positive distributions. J. Multivariate Anal. 10, 467-498.

KARlin, S. and Rubin, H. (1956a) Distributions possessing a monotone likelihood ratio. J. Amer. Statist. Assoc. 51, 637-643.

KARLIN, S. and RUBIN, H. (1956b) The theory of decision procedures for distributions with monotone likelihood ratio. Ann. Math. Statist. 27, 272-299.

LE CAM, L. (1996) Comparison of experiments-a short review. In Statistics, probability and game theory, volume 30 of IMS Lecture Notes Monogr. Ser., 127-138. Inst. Math. Statist., Hayward, CA. URL http://dx.doi.org/10.1214/lnms/1215453569.

Lehmann, E. L. (1952) Testing multiparameter hypotheses. Ann. Math. Statistics 23, 541-552.

Lehmann, E. L. (1955) Ordered families of distributions. Ann. Math. Statist. 26, 399-419.

Lehmann, E. L. (1988) Comparing location experiments. Ann. Statist. 16, 521-533.

Lehmann, E. L. and Rojo, J. (1992) Invariant directional orderings. Ann. Statist. 20, 2100-2110.

LEVIN, J. (2001) Information and the market for lemons. Rand J. Econom. 32, 657-666.

Müller, A. and Stoyan, D. (2002) Comparison methods for stochastic models and risks. Wiley Series in Probability and Statistics. John Wiley \& Sons Ltd., Chichester.

PERSICO, N. (2000) Information acquisition in auctions. Econometrica 68, 135-148.

Quah, J. K.-H. and Strulovici, B. (2009) Comparative statics, informativeness, and the interval dominance order. Econometrica 77, 1949-1992.

Shaked, M. and Shanthikumar, J. G. (2007) Stochastic orders. Springer Series in Statistics. Springer, New York. URL http://dx.doi.org/10.1007/978-0-387-34675-5.

Stein, C. (1951) Notes on a Seminar on Theoretical Statistics. I. Comparison of experiments. University of Chicago.

StRASSEN, V. (1965) The existence of probability measures with given marginals. Ann. Math. Statist. 36, 423-439.

Torgersen, E. (1991) Comparison of statistical experiments, volume 36 of Encyclopedia of Mathematics and its Applications. Cambridge University Press, Cambridge.

WhitT, W. (1982) Multivariate monotone likelihood ratio and uniform conditional stochastic order. J. Appl. Probab. 19, 695-701. 